\title{
Listening to the Deep: Live monitoring of ocean noise and cetacean acoustic signals
}

\author{
M. André*, M. van der Schaar, S. Zaugg, L. Houégnigan, A.M. Sánchez, J.V. Castell \\ Laboratori d'Aplicacions Bioacústiques, Universitat Politècnica de Catalunya (UPC), Centre Tecnològic de Vilanova i la Geltrú, Avda. Rambla Exposició, s/n, \\ 08800 Vilanova i la Geltrú, Barcelona, Spain
}

\section{A R T I C L E I N F O}

\section{Keywords:}

Noise pollution

Passive acoustic monitoring

Cetaceans

Deep-sea observatories

Noise mitigation

\begin{abstract}
A B S T R A C T
The development and broad use of passive acoustic monitoring techniques have the potential to help assessing the large-scale influence of artificial noise on marine organisms and ecosystems. Deep-sea observatories have the potential to play a key role in understanding these recent acoustic changes. LIDO (Listening to the Deep Ocean Environment) is an international project that is allowing the real-time longterm monitoring of marine ambient noise as well as marine mammal sounds at cabled and standalone observatories. Here, we present the overall development of the project and the use of passive acoustic monitoring (PAM) techniques to provide the scientific community with real-time data at large spatial and temporal scales. Special attention is given to the extraction and identification of high frequency cetacean echolocation signals given the relevance of detecting target species, e.g. beaked whales, in mitigation processes, e.g. during military exercises.
\end{abstract}

(c) 2011 Published by Elsevier Ltd.

\section{Introduction}

The sea environment has always been filled with noise (from animals and physical processes), although the last hundred years have seen the introduction of many anthropogenic sources that are currently contributing to the general noise budget of the oceans. The extent to which noise in the sea impacts and affects marine ecosystems has become a topic of considerable concern to the scientific community. Anthropogenic noise, including acoustic signals necessary to study the marine environment, can interfere with the natural use of sound by sea organisms. For geophysicists, seismologists and oceanographers, sound is the most powerful tool available to determine the geological structure of the seabed and to look for oil and gas reserves deep below the seafloor. As far as defense is concerned, sound is also used to detect long-range targets. On the other hand, unnecessary or unintentional noise sources, i.e. sources that are associated to specific activities but contain no information (shipping for instance) are constantly introduced in the marine environment.

The question is whether human-generated noise may interfere with the normal use of sound by the marine animals (i.e. chronic effects that may affect the long-term ability of marine animals to develop their normal activities, reproduce, and maintain sustainable populations) or cause physical harm to them (i.e. acute effects that may compromise the short-term ability of these animals to survive).

\footnotetext{
* Corresponding author.

E-mail address: michel.andre@upc.edu (M. André).

URL: http://www.lab.upc.es (M. André).
}

Sound is a primary means by which many marine organisms learn about their environment and is also, for many species of marine mammals and fish, the primary means of communicating, navigating, and foraging. Biological sounds are equally diverse as anthropogenic sources and can be emitted intentionally or unintentionally by numerous organisms. Unintentional sounds could comprise those produced by schools of fish swimming through the ocean or the release of air by large groups of fish as they adjust their buoyancy. Intentional sounds, including whale calls, dolphin echolocation signals, and fish vocalizations, are believed to be produced in various species for communication, echolocation, and perhaps even acoustic imaging of their environment.

To help managing the marine ecosystem environment and mitigating adverse effects of anthropogenic noise, there is a need to understand more about the role of sound production and reception in the behavior, physiology, and ecology of these species and to provide insights into important aspects of their biology. This understanding, in turn, previously requires the detection and identification of the sound sources of interest under real conditions.

Many cetacean species can be identified by their specific calls. The recording of these signature acoustic signals can reveal their presence in monitored areas. Since sound propagates efficiently in water, the detection range of these signals can be quite large, exceeding $100 \mathrm{~km}$ in favorable conditions for low-frequency calls (e.g. Stafford et al., 1998; Simard et al., 2008), far above visual detection methods. This acoustic potential to non-intrusively detect and monitor cetacean species in their environment gave rise to passive acoustic monitoring (PAM) techniques, for which research is very active (review by Mellinger et al., 2007) as reveals the series of biennale international workshops dedicated to this 
rapidly evolving field since 2003 (Desharnais, 2004; Adam, 2006; Moretti et al., 2008; Pavan, 2009). Localizing whales from their sounds in their habitats was initiated by Watkins and Schevill (1972) in the 1970s. This was rapidly applied to tracking whales over large distances (e.g. Cummings and Holliday, 1985; Clark et al., 1986). Advances in electronics, computers and numerical analysis now make this PAM technology more accessible and affordable to small research budgets. Various systems have been used, including radio-linked systems, drifting buoys, and arrays of autonomous recorders for versatile and long-term deployments (c.f. Refs. in Simard et al., 2008). The goal of such PAM systems, is the continuous mapping of presence and distribution of whales over ocean basins (e.g. Greene et al., 2004; Sirovic et al., 2007; Stafford et al., 2007; Mas et al., 2008) and assessing their densities, (e.g. Ko et al., 1986; McDonald and Fox, 1999; Clark and Ellison, 2000), sometimes in quasi real-time (e.g. Tiemann and Porter, 2004; André et al., 2005, 2007a,b; Delory et al., 2007). Their performance in effectively accomplishing these tasks depends on the characteristics of the targeted cetacean acoustic signals, the environment, the type of equipment used, its deployment and configuration. This performance may significantly vary from case to case.

However, in any case, PAM's success first depends on the capacity to isolate the target signals from the rest of sounds in which they are embedded, especially for distant sources and low signal to noise ratios (SNR). The acoustic signal source level, propagation loss, and local background noise levels determine detection ranges (c.f. Sirovic et al., 2007; Stafford et al., 2007; Simard et al., 2008). Moreover, cetacean sounds vary considerably in time-frequency, from infrasonic calls of baleen whales to ultrasonic clicks of toothed whales, and in amplitudes among species and within a species' vocal repertoire (e.g. André and Kamminga, 2000; van der Schaar et al., 2007a,b; Mellinger et al., 2007). Ocean noise level also exhibits considerable variability in space and time, caused by fluctuating natural sources, such as wind, ice, rain, sounds produced by various organisms, and anthropogenic sources such as shipping (c.f. review NRC, 2003). Sound speed structures over the water column can focus sounds from distant sources into sound channels. The 3D spatial arrangements of the sources and the hydrophones, their depth relative to the sound channel are therefore relevant to the PAM configuration.

In addition to the development and broad use of PAM techniques, another challenge is to obtain long-term access to data for the assessment of the large-scale influence of artificial noise on marine organisms and ecosystems. Understanding the link between natural and anthropogenic acoustic processes is indeed essential to predict the magnitude and impact of future changes of the natural balance of the oceans. Deep-sea observatories have the potential to play a key role in the assessment and monitoring of these acoustic changes.

The Laboratory of Applied Bioacoustics (LAB) is currently leading an international project titled "Listen to the Deep Ocean Environment (LIDO)" (LIDO, 2008) to apply and extent developed techniques for passive acoustic monitoring to cabled deep sea platforms and moored stations (André et al., 2008). The software framework, called S-SONS, is currently active at the ANTARES (http://antares.in2p3.fr/) neutrino observatory, the OBSEA (http:// www.obsea.es) shallow water test site, the NEPTUNE Canada (http://www.neptunecanada.ca/) observatory, the JAMSTEC (http://www.jamstec.go.jp/e/) network of underwater observatories and at the NEMO (http://nemoweb.lns.infn.it/) site after the observatory has been redeployed. Part of the system is being tested for suitability on autonomous gliders in collaboration with the NURC (Dassatti et al., 2011). The software contains several independent modules to process real-time data streams. Among these, there are dedicated modules for noise assessment, detection, classification and localization. To summarize the LIDO system, it takes as input an acoustic data stream and produces as output the characterization of the acoustic events that were detected in the data (written to an XML file), spectrograms for quick visualization and compressed audio. These outputs are then made available on the Internet where they can be viewed with a specific application. The public interface can be found at http://www.listentothedeep.net. It should be noted that the compressed audio is provided to allow users to listen to a sound stream with minimal bandwidth usage; but it is specifically not intended for scientific analysis. The raw data is optionally stored locally if there is an interest in subsequent research.

Here we present the LIDO real-time acoustic data concept and management, illustrated by several results from the above observatories, with a special attention given to the extraction and identification of cetacean echolocation signals (ultrasonic cetacean clicks), including beaked whales' and delphinids', noise from shipping and sperm whale clicks, for their relevance in mitigation processes.

\section{Objectives}

Through the use of the information provided by the existing and future underwater observatories where the automatic detection, classification and localization (DCL) processes are applied, the purpose of the LIDO framework is to (1) evaluate the human and natural contributions to marine ambient noise and describes the long-term trends in ambient noise levels, especially from human activities; (2) outline the research needed to evaluate the impacts of ambient noise from various sources (natural, biological, commercial, offshore, and acoustic-based ocean research) on marine species, especially in biologically sensitive areas; (3) review and identify gaps in existing marine noise databases and recommend research needed to develop a model of ocean noise that incorporates temporal, spatial, and frequency-dependent variables.

Although LIDO concentrates primarily on the effects of noise on marine mammals, it also considers other species as well (e.g. fish) that are part of the ecosystem and food web on which marine mammals depend. The frequency band to be studied ranges from 1 to $200,000 \mathrm{~Hz}(200 \mathrm{kHz})$ depending on the acoustic sensor characteristics, since this is the entire bandwidth that various marine organisms are capable of detecting. An important requirement for the LIDO system is that measurements and analysis results are obtained in real-time, or at least without the need to physically recover submerged hardware. The results are then readily available for mitigation procedures when monitoring is performed in an area with active sea-operations such as construction or ocean acoustic tomography.

The following key issues are considered to be essential to achieving these objectives and to evaluating the human and natural contributions to ocean noise:

- to develop quantitative relationships between man-made noise and levels of human activity;

- to establish a long-term ocean noise monitoring program covering, depending on hydrophone sensitivity, the frequency band from 1 to $200,000 \mathrm{~Hz}$;

- to conduct research on the distribution, migration patterns, characteristics, identification and classification of marine biological sounds and organisms, especially marine mammals;

- to monitor ocean noise in geographically diverse areas with emphasis on marine mammal habitats and allow comparison at large temporal and spatial scales;

- to examine the impact of ocean noise on nonmammalian species in the marine ecosystem. 
The design and implementation of research on the effects and control of man-made noise in the marine environment must be an interdisciplinary enterprise. Contributions and expertise are needed from electronics experts on the choice and calibration of transducers for monitoring natural, biological and anthropogenic sound sources, from physical acousticians to process signal/information provided by the observatories, from marine biologists to identify species sound-related behavior and seasonality and large scale data, from psychoacousticians to assess species related hearing sensitivities and from statisticians for the initial design, data analysis and presentation.

\section{System description and performance}

\subsection{Automated real-time monitoring of acoustic events and noise}

PAM has the potential to be implemented continuously and over long time periods, resulting in large and representative datasets. However, this inevitably leads to a high rate of audio data acquisition that could be problematic when the data need to be transmitted, stored, or analyzed. For observatories with a limited power supply or bandwidth, e.g. radio-linked autonomous buoys, transmission, storage, or additional data processing (e.g. automated classification, data compression) has to be optimized, which may imply the loss of potentially interesting information. For cabled observatories where power and communication are not an issue, limitations arise with storage. In any case, on the one hand, there is a need for immediate mitigating actions when facing potentially harmful acoustic events; on the other hand, there is a necessity for long-term monitoring of noise. This calls for the development of a robust technique that provides both historical statistical data on noise and alarms on specific acoustic events, i.e. a fully automated real-time detection and classification system that provides this information while minimizing technical costs (storage, computation time). The approach proposed here divides the recording bandwidth in frequency bands that cover the acoustic niche of most species and applies a series of detectors and classifiers. The information from the detection and classification modules is then used by localization and tracking algorithms in order to monitor the presence and activity of cetacean species. This allows assessment of the short-, medium, and long-term contributions of noise sources in these acoustic niches (André et al., 2010; Houégnigan et al., 2010; Zaugg et al., 2010).

The detection module contains triggers for short transient sounds (such as echolocation signals from toothed whales, but also cavitation sounds from ship propellors or explosions from air guns), short tonal signals (such as dolphin whistles, depth sounders, calls from baleen whales) and constant tonal sounds (constant frequencies that can be caused by artefacts from electrical interference or shipping traffic). Other characteristic sounds that are not covered by these detectors are being added when encountered at platforms.

The classification module is built to function on the output of the detector. Currently, it operates on transient sounds. The focus is on transients since these signals can come from a much larger number of different sources than other types of signals. When detected, transients are isolated from the data stream and a number of features are extracted (detailed below). The features are used as inputs to a neural network classifier. Based on training sets, radial basis function networks are created capable of distinguishing between classes.

The localization module is specifically designed to be used with small hydrophone arrays, such as those that are available at NEMO or ANTARES, and performs localization on detected transient sounds. Due to the array limitations, it primarily computes a bearing estimate. Range estimates are under consideration based on tracking a particular classified source with known source levels, but these are not yet implemented. As the system needs to operate in real-time, not all transients that are detected are always processed; especially shipping traffic can create large numbers of impulses in a short time period. Priority is given to signals that were classified and originated from a source of interest.

\subsection{The LIDO DCL architecture}

Fig. 1 shows an overview of the processing done in the LIDO framework. Phase 1 and 2 are preliminary steps that must ensure the real-time process of the data. In undersea recordings background noise is always present (sea noise). The first phase of the system detects broad classes of events. The second phase classifies segments into more specific classes. Most of the time this sea-noise presents little interest but fills terabytes of unnecessary storage. The challenge is here to be able to find a reliable set of filters or detectors able to extract the interesting information that would be included with the background-noise, like dolphin calls and sonar, sperm and beaked whale clicks, or noise produced by ship engine. A detector is an algorithm that accepts a segment of acoustic data as input and gives a single number as output. The output number is usually designed such that (1) it is equal or close to zero if only sea-noise is present in the segment (2) it takes larger values if an additional signal is present. By applying a threshold on the output number, the detector can take a decision, that is: automatically label the segments as "sea-noise-only" vs. "presence of interesting signal". The concept of the broad categories returned by Phase 1 is to narrow the amount of data supplied to the more sophisticated algorithms of Phase 2. These Phase 2 algorithms are slightly slower. Hence, narrowing the input in quantity alleviates the slowness and improves the overall robustness by having a well-defined input.

The system's modules can be run independently, thereby allowing to implement smaller and faster versions, aimed at specific classes of sounds or mitigation scenarios, like the detection of beaked whales and delphinid species. New modules can be incorporated into the system.

Although LIDO considers the whole bandwidth and a series of detectors and classifiers (see Sections 3.4 and 3.5), this paper especially pays attention to the extraction of ultrasonic cetacean clicks (UCC), belonging to beaked whales and delphinids from sperm whale clicks (SWC) and Impulsive Ship Noise (ISN). The frequency bands of interest here are the following (assuming a $96 \mathrm{kHz}$ sampling rate):

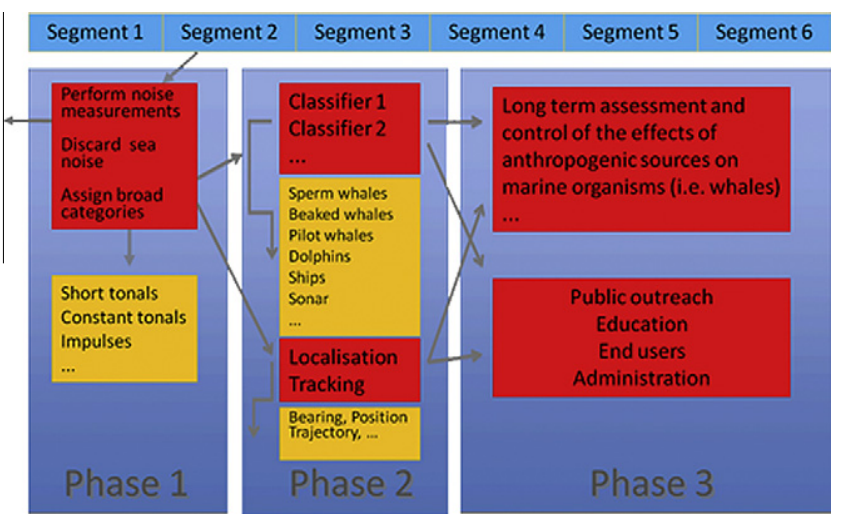

Fig. 1. Overview of the LIDO analysis concept. 


\subsubsection{1-5 and 5-20 kHz bands}

Impulsive Ship Noise, broadband shipping noise and ambient noise are generally more intense at lower frequencies and can mask some other impulse sources. A better detection will be performed in the band $5-20 \mathrm{kHz}$. However, distant/off-axis sperm whale clicks have most energy below $5 \mathrm{kHz}$ and would be better detected in the band $1-5 \mathrm{kHz}$.

\subsection{2. $20-46 \mathrm{kHz}$ band}

This band is aimed at detecting ultrasonic impulses (e.g. beaked whales and delphinid sonar).

\subsection{Noise measurement module}

The noise measurement module computes statistics on fixed length intervals, such as sound maxima, RMS level and third octave levels, especially following the recommendations of the European Marine Strategy Framework Directive (2008/56/EC). In particular, the Descriptor 11.1 (Tasker et al., 2010) focuses on high amplitude impulsive anthropogenic sound within a frequency band between $10 \mathrm{~Hz}$ and $10 \mathrm{kHz}$, assessed using either sound energy over time (Sound Exposure Level SEL) or peak sound level of the sound source, while the Descriptor 11.2 addresses background noise without distinguishable sources that can lead to masking of biological relevant signals, alter communication signals of marine mammals, and through chronic exposure, may permanently impair important biological functions. This latter indicator requires a set of sound observatories to enable trends in anthropogenic background noise to be followed (noise within the $1 / 3$ octave bands 63 and $125 \mathrm{~Hz}$, center frequency).

Fig. 2 shows an example of noise measurements conducted during a full day at the ANTARES observatory (RMS level of the whole bandwidth, and in the bands $1-5,5-20,20-110 \mathrm{kHz}$ ) allowing to extract the contribution of sources in different bandwidths, and their relationship with the presence of sperm whales and dolphins during the same time period.

\subsection{Detection module}

Detection of acoustic events are handled by two different algorithms, one for impulsive sounds and one for short tonal sounds. Details on these algorithms are described in Zaugg et al. (2010).
The impulse detector processes the data in a configurable number of frequency bands: e.g. $0-500 \mathrm{~Hz}, 500-1,1-5,5-20 \mathrm{kHz}$, above $20 \mathrm{kHz}$. This separation is used to allow different configuration parameters specifically designed to trigger on longer low frequency or shorter high frequency impulses, covering from baleen whale calls to beaked whale ultrasonic clicks. The left image in Figs. 3 and $5 c$ show example detections obtained in the band above $20 \mathrm{kHz}$ on ultrasonic cetacean echolocation signals (single clicks and buzzes) and Fig. 5(a-c) show detections obtained in the same band on sperm whale clicks and shipping impulses.

The short tonal detector is configured to detect whistle like sounds from dolphins or tonal calls produced by various baleen whale species. For the LIDO system detection is usually done in three different frequency bands: below $5,2-20 \mathrm{kHz}$ and above $20 \mathrm{kHz}$. The lowest band is aimed at baleen whales, the middle band at dolphins (including orcas). The highest band has not been triggered by biological events so far, but it can be triggered by specific anthropogenic sources, such as the line localization echo sounder at ANTARES. The right image in Fig. 3 shows detection on vocalizations of a humpback whale. On the various platforms where the LIDO system has been running this detection procedure has so-far been sufficient as there were not many other sources that produce these particular sounds. The detector is configured in such a way that it almost functions as a classifier by itself (e.g. Fig. 3). As an example, at the ANTARES site, the echo sounder that is used for localization of the lines often triggers the short tonal detector. As these are in frequencies over $20 \mathrm{kHz}$, they are easily separated from dolphin whistles, which have a specific detector operating in the range between 2 and $20 \mathrm{kHz}$.

For real-time consideration, both algorithms operate in constant time, regardless of the number of detections that are encountered. The execution time does depend on the bandwidth that the detector operates on. The currently implemented version evaluates all detections (4-impulse and 3-short tonal bandwidths) in a $22 \mathrm{~s}$ segment of data sampled at $96 \mathrm{kHz}$ in under a second on a single core from an Intel XEON X3360 $(2.8 \mathrm{GHz})$.

\subsection{Classification module}

For feature extraction and classification of the sources a more complex approach was adopted (Fig. 4). The current classification algorithm that is used in the LIDO system is a continuation of an
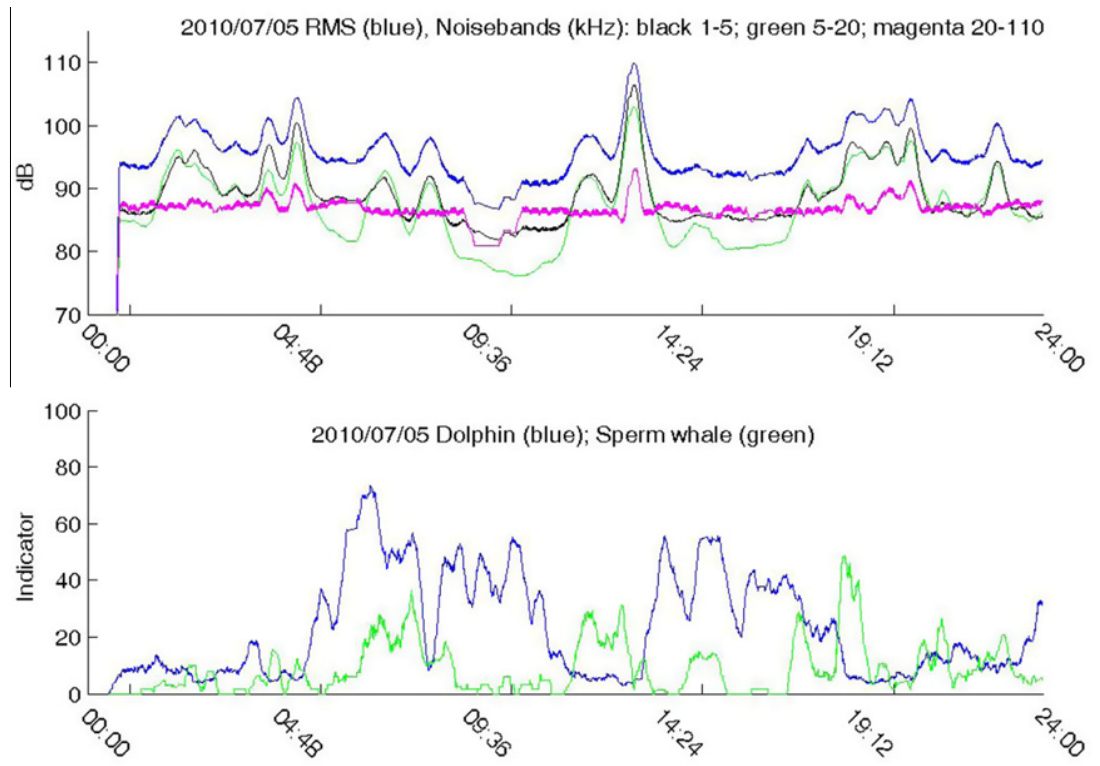

Fig. 2. Noise measurements and indicators of sperm whale and dolphin presence at the ANTARES observatory on May the 7th, 2010. 

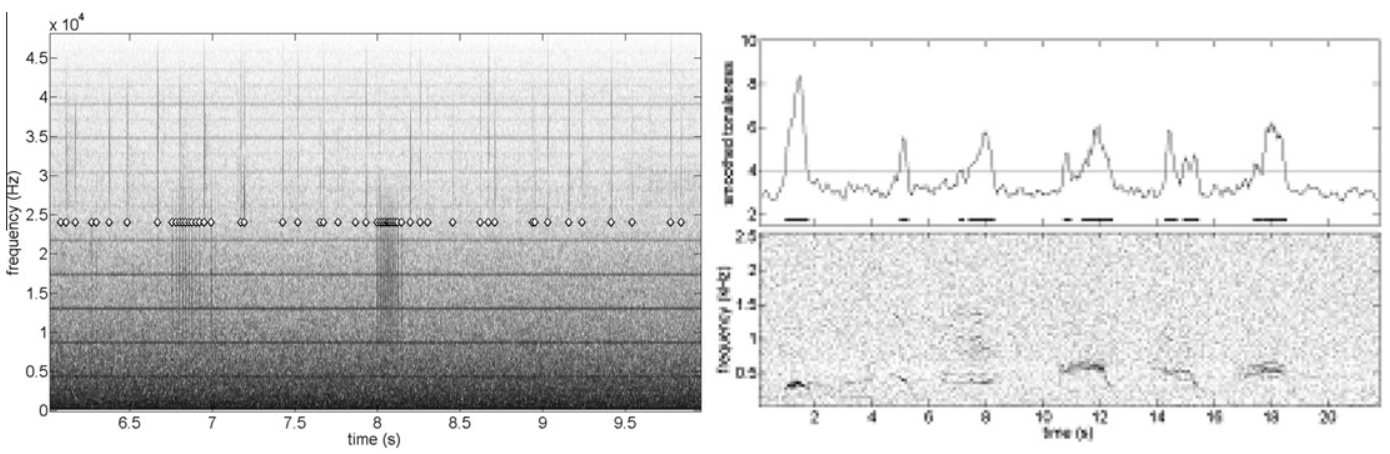

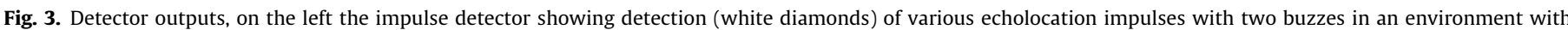
interference lines; on the right the short tonal detector acting on humpback whale vocalizations (detected regions are shown as horizontal black bars).

algorithm described in Zaugg et al. (2010). This proposal suggests defining classes for sperm whale echolocation signals, ultrasonic cetacean impulses (beaked whale and dolphin echolocation signals), shipping impulses, dolphin whistles and baleen whale vocalizations. More classes may be added on demand, but to allow generalization it requires a set of training data of sufficient quality.

A short piece of audio $(21 \mathrm{~ms})$ centerd on the impulses that are detected in Phase 1 is extracted and used to extract features. In this process, impulses are characterized by their temporal and spectral shape (noted $E\left(x_{i}\right)$ where $x_{i}$ represents either time-bins or frequency-bins) with the following descriptors:

A measure of location : $m_{1}=\sum_{i=1}^{n} x_{i} E\left(x_{i}\right)$

A measure of dispersion : $\quad m_{2}=\sqrt{\sum_{i=1}^{n}\left(x_{i}-m_{1}\right)^{2} E\left(x_{i}\right)}$

A measure of asymmetry : $m_{3}=\frac{1}{m_{2}^{3}} \sum_{i=1}^{n}\left(x_{i}-m_{1}\right)^{3} E\left(x_{i}\right)$

A measure of density : $m_{4} \frac{1}{m_{2}^{4}} \sum_{i=1}^{n}\left(x_{i}-m_{1}\right)^{4} E\left(x_{i}\right)$

A measure of peakiness : $\quad p=-\frac{1}{\log (n)} \sum_{i=1}^{n} E\left(x_{i}\right) \log \left(E\left(x_{i}\right)\right)$

These descriptors are evaluated both in the time and frequency domains leading to nine features (location is not used for the temporal shape). In addition, sequences of transients are characterized by inter-pulse intervals, as some sources can produce much more stable sequences than others.

Then, the features obtained for an impulse enter an RBF neural network and give as output: $\mathrm{p}(\mathrm{SWC}), \mathrm{p}(\mathrm{ISN})$ and $\mathrm{p}(\mathrm{UCC})$, which are the estimated probabilities of being a SWC, ISN or UCC. The computation of the features takes a small amount of time, but much less than the time necessary for the detection modules. Classification as it runs on current LIDO servers can be considered instantaneous.

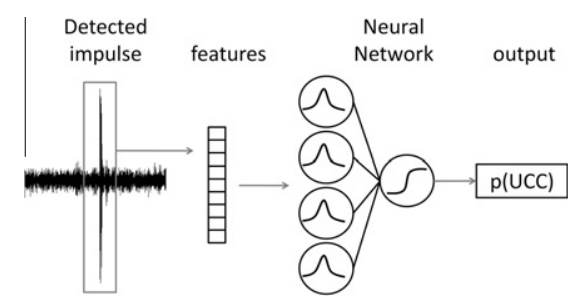

Fig. 4. Overview of the classification procedure applied to ultrasonic cetacean clicks. A short frame centerd on a detected impulse is automatically identified by a detector stage. This frame is used to extract nine features. These features are passed to a trained RBF neural network, which returns the estimated probability that the impulse is an ultrasonic cetacean click, short p(UCC).

\subsubsection{Assessment of classification performance}

The detection and classification at the level of individual impulses are illustrated with examples. The plots in Fig. 5, show spectrograms of recordings with (Fig. 5a) impulses from ship noise (ISN), (Fig. 5b) sperm whale clicks (SWC) and (Fig. 5c) a regular train of ultrasonic cetacean clicks (UCC) embedded in ISN. In a first phase, detection was performed in the band $20-46 \mathrm{kHz}$. The detected position of impulses is shown as black crosses (arbitrarily plotted at $24 \mathrm{kHz}$ ). In a second phase, the automated assignment of $\mathrm{p}$ (UCC) was obtained for each detected impulse via a neural network. The values of $\mathrm{p}(\mathrm{UCC})$ are represented by the vertical position of the white dots (plot floor: $\mathrm{p}(\mathrm{UCC})=0$, plot ceiling: $\mathrm{p}(\mathrm{UCC})=1$ ). Many of the ISN and SWC had energy above $20 \mathrm{kHz}$, hence they were detected in the first phase. The second phase processing by the neural network recognized true UCCs by assigning them values of $\mathrm{p}$ (UCC) close to 1 while most of the SWCs and ISN were assigned values close to 0 . The final output, $N_{\mathrm{Ucc}}$, which represents a whole segment of $22 \mathrm{~s}$, was obtained via a weighted sum of the $\mathrm{p}(\mathrm{UCC})$ that were larger than 0.5 .

Shown in Fig. 6 are the results of the detection and classification of impulses at the level of $22 \mathrm{~s}$ segments. These results were obtained on data from the NEMO-ONDE deep-sea observatory (Migneco et al., 2008; Riccobene et al., 1995), which is bottom mounted at $-2000 \mathrm{~m}$ depth east of the Sicilian coast. A neural network was trained with a data set recorded in May and tested with a data set recorded in August 2005 (a) and vice versa (b). Each dot represents a segment of $22 \mathrm{~s}$ of audio containing almost exclusively impulses from one of the three classes: impulses from ship noise (ISN, squares), sperm whale clicks (SWC, circles) and ultrasonic cetacean clicks (UCC, triangles). The class UCC includes clicks from delphinids and beaked whales. Upper subplots: the number of detected impulses $\left(N_{\text {det }}\right)$ in the $20-46 \mathrm{kHz}$ band is shown. Many ISN and SWC and all but on UCC segments triggered this detector. Lower subplots: the final output is $N_{\mathrm{Ucc}}$, the estimated number of UCCs per segment. When a threshold was set at a value of 2 (gray line), the percentage of correctly detected UCC segments from all UCC segments was $97 \%$ and $100 \%$, respectively for test data from August (a) and May (b). The false detection rate, that is, the percentage of detected non-UCC segments from all non-UCC segments was $6 \%$ and $17 \%$, respectively. A a posteriori scrutiny of non-UCC segments with a $N_{U C C}>2$ revealed that most contained UCCs that had been missed during manual assignment of the segments to the three classes. The resulting corrected false detection rate was $2 \%$ and $1 \%$, respectively.

\subsection{Localization module}

In principle, precise source localization requires detailed knowledge of the marine environment (sea state, sound speed profile, 

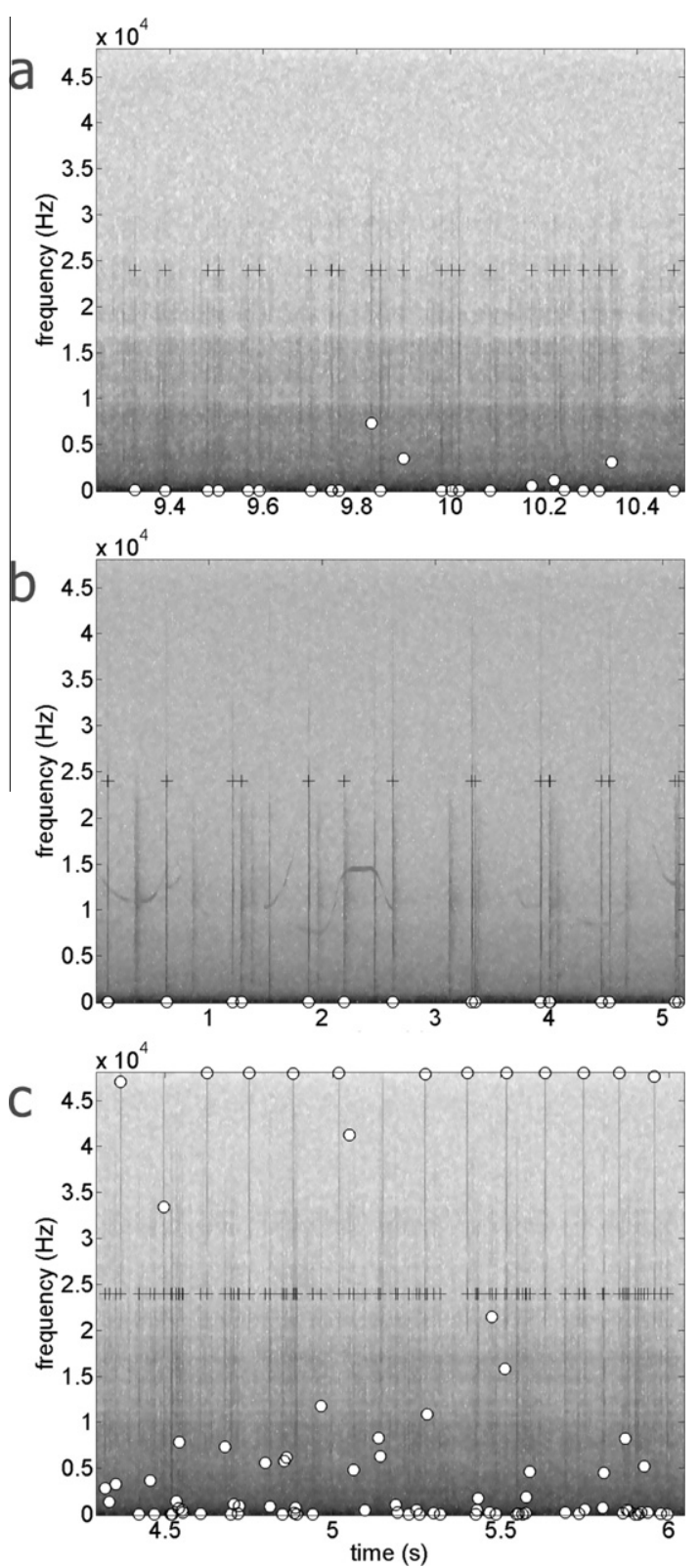

Fig. 5. Detection and classification of UCCs, SWCs and ISNs (see text for explanation of symbols).

etc.) and hydrophone array configuration (calibration, position, dimensions with regard to the frequency content of the signals of interest, etc.). Where detection and classification can be generalized to work on most archived data (perhaps with the help of a filter that is applied before analysis to remove specific sound characteristics that complicate the analysis), the multichannel and space-time nature of localization protocols create additional difficulties. When the LIDO system is used in combination with compact hydrophone arrays, the primary objective is to compute a real-time estimate of the bearing of a source. Once more information becomes available about the source (e.g. the class, the track), a range indication can be made based on the received signal, source level estimates and the track.

The algorithms used for bearing estimation in the LIDO system are described in Houégnigan et al. (2010). High real-time performances were obtained with the steered response power algorithm (space-time correlation between hydrophones) in combination with the generalized cross correlation (ROTH or SCOT filters).
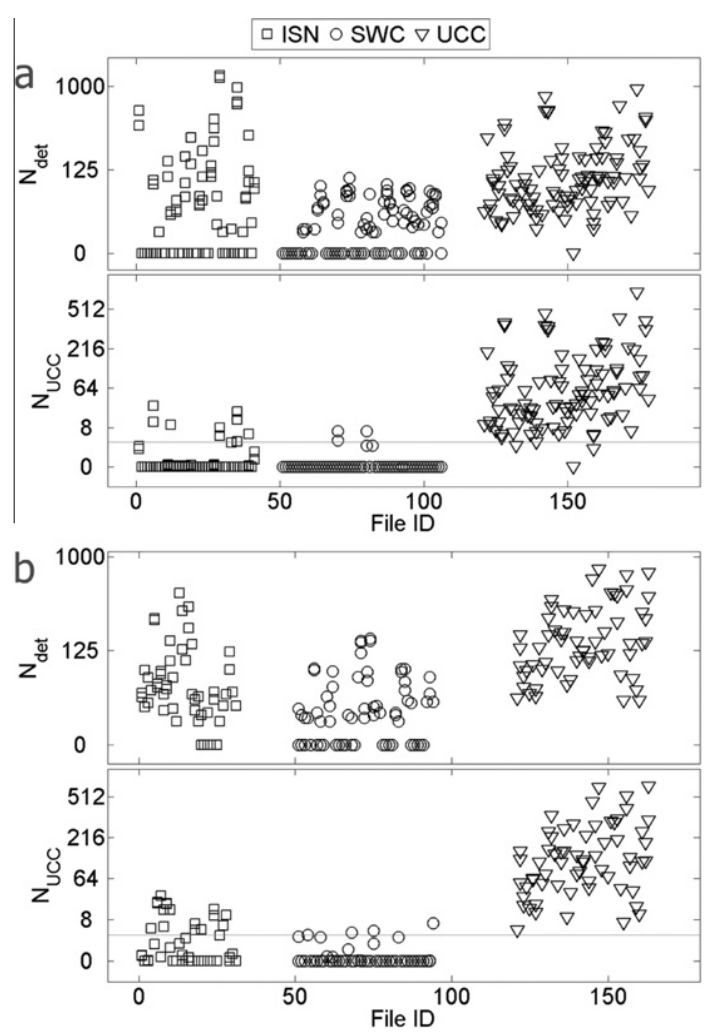

Fig. 6. Segment-level classification after the detected acoustic events entered the RBF neural network (see text for additional explanations).

Fig. 7 is reproduced from Houégnigan et al. (2010) and shows localization of an example impulse, a sperm whale track with slowly varying azimuth and the track of a ship where the azimuth changes much faster (sources were manually identified).

The latest implementation of this module was optimized for tetrahedral arrays and more generally for a compact array, but it can also provide bearing estimations for arrays with less than four hydrophones. The performance of the algorithm being linked to the number of hydrophones and array geometry, the quality of the estimation has to be evaluated case by case, for example in that case, e.g. a single line towed array will providing bearing estimates with a lesser accuracy.

A current implementation of the SRP algorithm combined with a SCOT filter processes 50 impulses in under $2 \mathrm{~s}$ on the same system as described in the detection section. In a real-time situation the number of impulses that are localized are limited by setting a maximum number of seconds that the algorithm is allowed to run. When there are many impulses, preference will be given to classified biological impulses.

\section{Discussion and conclusion}

\subsection{Real-time detection and classification of cetacean clicks}

The classification of ultrasonic cetacean clicks showed that many segments with sperm whale clicks and impulsive shipping noise did not trigger the high frequency detector at all. This is because these two classes of impulses often do not carry energy above $20 \mathrm{kHz}$. On the other hand, almost all segments with ultrasonic cetacean clicks were clearly detected. Hence, the detection stage allowed for a significant reduction in the data volume passed to the subsequent classifiers by selecting only segments with potential ultrasonic cetacean clicks. It is also clear that this detector 

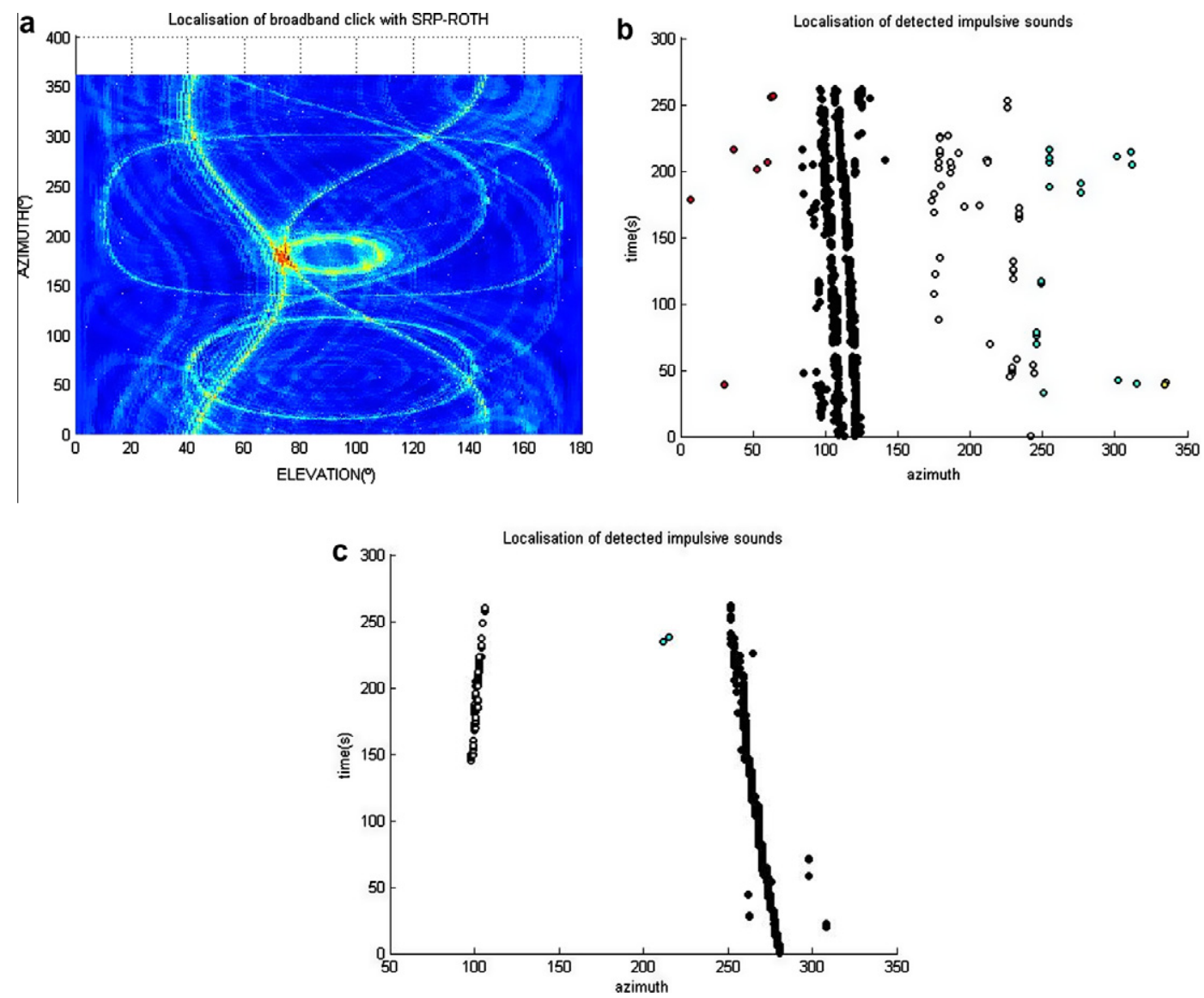

Fig. 7. Left image shows a bearing estimation on an impulse using the ROTH filter. Center and right image show tracks of a sperm whale and ship, respectively.

is not suitable to detect sperm whale clicks or impulsive shipping noise, since it processes the band $20-46 \mathrm{kHz}$. For these two classes, similar detectors and classifiers have been developed in the band $1-20 \mathrm{kHz}$.

The separation of ultrasonic cetacean clicks from sperm whale clicks and impulses from ship noise is an easy task for a human operator due to the higher frequency location of the ultrasonic cetacean clicks. The interest of the presented modules lie in their ability to perform this classification in a fully automated way with a very high accuracy and to work reliably over time periods of at least several months.

The detection stage returned an accurate estimation of the impulse's location. We showed here that this estimated location can be effectively used by the second stage classifiers. The automated location could also be directly used by other second stage algorithms. For example, by sequence analysis procedures that automatically estimate the inter click interval of regular click trains, which could enable to draw conclusions on the taxonomic group that emitted the clicks (e.g. delphinids, beaked whales).

The feature extraction performed before the classification is a computationally intensive step and should only be performed when necessary. Clicks from cetaceans are generally produced in relatively rapid trains and in a segment of $22 \mathrm{~s}$, we typically expect to find several dozens of clicks. Time can be saved by classifying only segments with a sufficiently large number of detected impulses. In this way segments with isolated detections will not be processed at all by the second stage modules.

The simplicity and modularity of the method enables it to be easily adapted to other classes of impulsive sounds. For example, the method has been used with success to separate sperm whale clicks from impulsive shipping noise. In the future, it could be adapted to separate cetacean species.

In a scenario of real time mitigation, for example during navy exercises or seismic surveys, the recorded data stream can be expected to be contaminated with a relatively constant background of impulsive shipping noise. The detection of cetacean echolocation clicks is especially important for taxa that produce little or no tonal calls such as beaked whales or the sperm whale. In this context, a real time detection method such as the one presented here represents a very promising option, since it is able to accurately detect ultrasonic cetacean clicks with a very small false detection of shipping impulses.

\subsection{Data distribution}

One of the problems with continuous data acquisition is that the data is not well accessible for research. Large amounts of data are recorded that have little interest, and often a research objective only includes a very specific acoustic event. Automatically scanning all data for this particular event is possible, but time consuming and requires all data to be copied or made accessible from stored hard disks or tape. Real-time monitoring and analysis as presented in this paper not only gives the opportunity to store those parts of the data that may be of interest for future research, leading to much smaller storage requirements, but also maintains a complete database of events that occurred in an area, which in turn gives immediate insight in the local acoustic conditions and allows those interested to directly select data that is of possible research interest (assuming that it was included for detection, otherwise a specific detection module can always be added to a monitoring platform). 
It is expected that the database will help building and understanding long-term trends in migration patterns, distribution and relationship with noise of large cetacean species.

\subsection{Underwater observatories}

The needs for a network of ocean observing systems cross many applied and research areas of earth and marine science. Many of the science areas that can be examined using such systems have direct impacts on societal health and well being and our understanding of ocean function in a changing climate. In particular, the European Seas Observatory NETwork (ESONET) Network of Excellence has been evaluating observatory design requirements, data management needs, standardization and interoperability, social implications, outreach and education, as well as financial and legal aspects of developing such a system. Additionally, several demonstration missions are being carried out, in part, to evaluate various technical options and the implementation of standardized data management approaches. Observatory networks have great potential to address a growing set of Earth science questions that require a broad and integrated network of ocean and seafloor observations. ESONET NoE activities are also importantly integrating researchers in the European Community, as well as internationally.

There is now wide recognition that research addressing science questions of international priority, such as understanding the potential impacts of climate change or geohazards like earthquakes and tsunamis, and the effects of noise pollution, should be conducted in a framework that can address questions across adequate temporal and spatial scales.

The LIDO (Listening to the Deep-Ocean Environment) objectives in terms of acoustic monitoring of noise and marine mammals directly converge to answer key questions on the evolution of the oceans. They offer a unique opportunity to redesign research protocols, optimize the analysis, give open access to long-term time series of data to the scientific community, reducing costs, improving our understanding of sound source interactions and mitigating adverse effects of anthropogenic noise.

LIDO modular architecture of real-time acoustic data management has been built to be easily adaptable not only to a great diversity of supports (cabled observatories, radio-linked autonomous buoys, towed arrays, gliders, ROVs, AUVs, etc.) but also to diverse situations and configurations that take into account changes of background noise, topography, oceanographic paramaters as well as the changing presence of marine mammal species in areas of interest.

\subsection{A mitigation tool for the offshore industry}

The LIDO acoustic detection, classification and localization (DCL) system can be integrated in a series of expandable radiolinked autonomous buoys that are timely deployed during offshore operations. In that case, the DCL is performed at buoy level. A mesh network allows buoy-to-buoy communication and an alert service provides the ship/offshore platform with the DCL analysis: the real-time continuous monitoring of cetacean presence.

The advantages are relevant:

- The LIDO DCL is automated and performed no matter sea state or light conditions.

- No expertise is needed onboard the survey vessels/offshore platforms since the alert service informs on the identification and position of cetacean species that is displayed on a userfriendly interface.

- The real-time continuous monitoring of cetaceans allows determining areas of exclusion depending on the sound source and the species involved.
- The decision-taking regarding the management of the offshore activity in presence of cetaceans falls under scientifically contrasted, objective and standardized procedures that ensure the sustainable development of the activity.

The LIDO contribution to the real-time assessment of noise interactions with cetaceans as well as to the long-term management of anthropogenic sound sources represents a step toward an improvement of the acoustic ecology status for marine organisms.

\section{Acknowledgments}

The authors want to thank the institutions that made possible the real-time acoustic data streams from the NEMO, ANTARES, NEPTUNE, and JAMSTEC observatories for their inclusion in the LIDO framework.

This study was partly funded by the European Commission under Contract ESONET 036851, EC FP6.

\section{References}

Adam, O. (Ed.), 2006. In: Proceedings of the 2005 Workshop on Detection and Localisation of Marine Mammals using Passive Acoustics. Appl. Acoust. 67 (1011).

André, M., Kamminga, C., 2000. Rhythmic dimension structures in Sperm Whale echolocation click trains: a function of identification and communication. J. Mar. Biol. Ass. UK 79, 1-7.

André, M., Johansson, T., Delory, E., van der Schaar, M., 2005. Cetacean biosonar and noise pollution. IEEE Oceans 2005 - Europe 2, Art. No. 1513199, pp. 1028-1032.

André, M., Johansson, A.T., Delory, E., van der Schaar, M., 2007a. Foraging on squid: the sperm whale mid-range sonar. J. Mar. Biol. Ass. UK 87, 59-68.

André, M., van der Schaar, M., Delory, E., Castell, J.V., Mas, A., 2007b. A passive acoustic solution to prevent collisions with whales. In: Proc. Fifth Int. Congress on Maritime Tech. Innov. and Res. Barcelona, 21-23 November, pp. 277-281.

André, M., Vallez, S., Gervaise, C., Sánchez, A., van der Schaar, M., Mas, A., Morell, M., 2008. Monitoring the ocean noise interactions. In: Proc. 22nd Conf. of the European Cetacean Society, The Netherlands.

André, M., Morell, M., Mas, A. et al., 2010. Best Practices in Management, Assessment and Control of Underwater Noise Pollution. Laboratory of Applied Bioacoustics, Technical University of Catalonia. CONAT150113NS2008029 (Available at <http://www.lab.upc.es>).

Clark, C.W., Ellison, W.T., 2000. Calibration and comparison of the acoustic location methods used during the spring migration of the bowhead whale, Balaena mysticetus, off Pt. Barrow, Alaska, 1984-1993. J. Acoust. Soc. Am. 197, 35093517.

Clark, C.W., Ellison, W.T., Beeman, K., 1986. Acoustic tracking of migrating bowhead whales. Oceans 86, 341-346.

Cummings, W.C., Holliday, D.V., 1985. Passive acoustic location of bowhead whales in a population census off Point Barrow. Alaska J. Acoust. Soc. Am. 78, 11631169.

Dassatti, A., van der Schaar, M., Guerrini, P., Zaugg, S., Houégnigan, L., Maguer, A., André, M. 2011. On-board Underwater Glider Real-Time Acoustic Environment Sensing. In: Proceedings of the OCEANS11, Santander, June 2011.

Delory, E., André, M., Navarro Mesa, J.-L., van der Schaar, M., 2007. On the possibility of detecting surfacing sperm whales at risk of collision using others' foraging clicks. J. Mar. Biol. Ass. UK 87, 47-58.

Desharnais, F. (Ed.), 2004. Proceedings of the 2003 Workshop on Detection and Localisation of Marine Mammals using Passive Acoustics. Can. Acoustics 32 (2).

Greene, C.R., McLennan, M.W., Norman, R.G., et al., 2004. Directional frequency and recording (DIFAR) sensors in seafloor recorders to locate calling bowhead whales during their fall migration. J. Acoust. Soc. Am. 116, 799-813.

Houégnigan, L., van der Schaar, M., Zaugg, S., André, M., 2010. Space-time and hybrid algorithms for the passive acoustic localisation of sperm whales and vessels. J. Appl. Acoust. 71, 1000-1010.

Ko, D., Zeh, J.E., Clark, C.W., et al., 1986. Utilization of acoustic location data in determining a minimum number of spring migrating bowhead whales unaccounted for by the ice-based visual census. Rep. Int. Whaling Comm. 36, $325-338$.

LIDO, 2008. Listening to the Deep-Ocean Sounds and Environment. European Commission, Contract ESONET, EC FP6.

Mas, A., van der Schaar, M., Morell, M., Alonso, J.-M., LLarena, M., Solé, M., André, M., 2008. Acoustic Map of the oceans: a tool for management. In: Proc. of the 22nd Conf. of the European Cetacean Society, The Netherlands.

McDonald, M.A., Fox, C.G., 1999. Passive acoustic methods applied to fin whale population density estimation. J. Acoust. Soc. Am. 15, 2643-2651.

Mellinger, D.K., Stafford, K.M., Moore, S.E., Dziak, R.P., Matsumoto, H., 2007. An overview of fixed passive acoustic observation methods for cetaceans. Oceanography 20 (4), 36-45 
Migneco, E. et al., 2008. Recent achievements of the NEMO project. Nucl. Instrum. Methods Phys. Res. A 588 (2008) 111-118.

Moretti, D., Mellinger, D., Desharnais, F. (Eds.), 2008. Proceedings of the 2007 Workshop on Detection and Localisation of Marine Mammals using Passive Acoustics. Can. Acoust. 36 (1)

NRC, 2003. Ocean noise and Marine Mammals. The National Academies Press, Washington, DC

Pavan, G., 2009. 4th International Workshop on Detection, Classification and Localization of Marine Mammals using Passive Acoustics. Università degli Studi di Pavia, Dept. of Animal Biology Centro Interdisciplinare di Bioacustica e Ricerche Ambientali.

Riccobene, G. et al., 1995. Long-term measurements of acoustic background noise in very deep sea. Nucl. Instrum. Methods Phys. Res. A 604 (2009), S149-S157.

Simard, Y., Roy, N., Gervaise, C., 2008. Passive acoustic detection and localization of whales: effects of shipping noise in Saguenay-St. Lawrence Marine Park. J. Acoust. Soc. Am. 123, 4109-4117.

Sirovic, A., Hildebrand, J.A., Wiggins, S.M., 2007. Blue and fin whale call source levels and propagation range in the Southern Ocean. J. Acoust. Soc. Am. 122, 12081215.

Stafford, K.M., Fox, C.G., Clark, D.S., 1998. Long-range acoustic detection and localization of blue whale calls in the northeast Pacific Ocean. J. Acoust. Soc. Am. 104, 3616-3625.
Stafford, K.M., Mellinger, D.K., Moore, S.E., Fox, C.G., 2007. Seasonal variability and detection range modeling of baleen whale calls in the Gulf of Alaska, 19992002. J. Acoust. Soc. Am. 122, 3378-3390.

Tasker, M.L., Amundin, M., André, M., Hawkins, A., Lang, W., Merck, T., ScholikSchlomer, A. Teilman, J., Thomsen, F., Werner, S, Zakharia, M. 2010. Marine strategy framework directive, task group 11, underwater noise and other forms of energy. In: Zampoukas (Ed.), JRC Scientific and Technical Reports. Office for Official Publications of the European Communities, p. 55. ISBN: 978-92-7915654-0.

Tiemann, C.O., Porter, M.B., 2004. Localization of marine mammals near Hawai using an acoustic propagation model. J. Acoust. Soc. Am. 115, 2834-2843.

van der Schaar, M. Delory, E., Català, A., André, M., 2007a. Neural network-based sperm whale click classification. J. Mar. Biol. Ass. UK 87 (1), 35-38.

van der Schaar, M., Delory, E., van der Weide, J., Kamminga, C., Goold, J.C., Jaquet, N., André, M., 2007b. A comparison of model and nonmodel based time-frequency transforms for sperm whale click classification. J. Mar. Biol. Ass. UK 87 (1), 27 34

Watkins, W.A., Schevill, W.E., 1972. Sound location by arrival times on a non-rigid three dimensional hydrophone array. Deep-Sea Res. 19, 691-706.

Zaugg, S., van der Schaar, M., Houégnigan, L., Gervaise, C., André, M., 2010. Real-time acoustic classification of sperm whale clicks and shipping impulses from deepsea observatories. Appl. Acoust. 71 (11), 1011-1019. 